(Mitteilung aus der Univ.-Augenklinik in Zürich. Direktor: Professor Dr. Sidler * Huguen in.)

\title{
Über die Veränderungen im Frühstadium der Retinitis exsudativa externa.
}

\author{
Von \\ Dr. Andreas Rados, \\ Assistent der Klinik. \\ Mit 6 Textabbildungen.
}

Die Erforschung der Retinalerkrankungen erbrachte in den letzten Jahren zwei Gruppen der Veränderungen der Netzhaut, die Angiomotosis retinae (v. Hippelsche Erkrankung) und die Retinitis exsudativa externa (Coatssche Erkrankung). Diese Erkrankungsformen liefern nach Beschreibung der Autoren gelegentlich klinische Bilder, wo die strenge Isolierung der beiden Formen nicht wohl möglich wäre. Fs ist nioht unser Zweok, bei dieser Gelegenheit beide Krankheitstypen zu behandeln, ersterer soll nur dermaßen erörtert werden, als es das Verständnis des zweiten erfordert.

Die von Hip pelsche Erkrankung wurde Klinisch schon im Jahre 1903 beschrieben, und v. Hi p peli) dachte selbst zunächst, daß ein Netzhantleiden tuberkulöser Herkunft vorliege. Der Prozeß war eigentlich nur an einer Arterie und der zugehörigen Vene lokalisiert, beide waren auf das Mehrfache des normalen Kalibers erweitert, stark geschlängelt und dunkelgefärbt. Beide erweiterten Gefäße verschwanden hinter einem peripheren, etwas prominenten, gelbweißen bzw. gelbroten, mehrere Papillen großen Herde. Zwischen diesem Herde und der Papille zeigte die Netzhaut eine langsam fortschreitende Abhebung. Blutungen spielten dabei keine Rolle, in einem Falle kam es nur zu hämorrhagischen Trübungen des Glaskörpers und gleichfalls zur Ansammlung von Cholesterinkrystallen im subretinalen Raum. Außerdem traten im Verlaufe der Gefäße weiße Fleckchen und an verschiedenen Stellen des Fundus weiße oder grawweiße, von Retinalgefäßen überquerte Flächen auf. Erst im Jahre 1911 war v. Hippel in die Lage gekommen, über anatomische Befunde berichten zu können. In der Zwischenzeit häuften sich ähnliche klinische Beobachtungen, und auch frühere Beobachtungen von Czermak, Goldzieher wurden als in diese Gruppe gehörige ausgesprochen. - $\checkmark$. Hippel fand eine Angiomatose der Netzhaut, der Tumor bestand, ähnlich wie in den beiden Augen des Patienten von $\left(z e r m a k^{2}\right.$ ), aus GefäBknäulen, welche in gewuchertes Gliagewebe eingelagert waren. Die Angiom.

1) v. Hi p pel, Ubber eine seltene Erkrankung der Netzhaut. Bericht der Ophthalm. Gesellsch. Heidelberg 59, 93, 1904 und Die anatomische Grundlage der von mir beschriebenen sehr seltenen Erkankung der Netzhaut. Dieses Archiv r9, 350. 1911.

2) Czermak, Path.*anat. Befunde bei der von E. $\checkmark$. Hip pel beschriebenen sehr seltenen Netzhauterkrankung. Bericht der OphthaIm. Ges. Heidelberg 1905. 
knoten saßen hauptsächlich in den inneren Netzhautschichten. v. Hippel erblickt das Charakteristische dieser Erkrankung in der Angiomatose, eine Auffassung, welche schon in der Benennung ausgesprochen wurde und seither sich in der Literatur allgemein eingebürgert hat. Viele Autoren bekennen diese Erklärungsmöglichkeit, obzwar in letzterer Zeit Meller' ${ }^{1}$ ) auf rein klinischen Überlegungen zu anderer Auffassung gekommen ist. Sein Fall bot klinisch ein ähnliches Bild wie das von $\nabla$. Hippel beschriebene. Frweiterung und Schlängelung eines GefäBpaares bei einem 30 jähr. Manne, graue Trübung und Verdickung der Netzhautperipherie, welche ungefähr das 5 fache der normalen Dicke darbot, weiter große runde rote Flecken, in die sich Gefäße einsenkten. Meller erblickt auch eine Tumorbildung, nur wird die Hauptveränderung der Gliawucherung beigelegt. Statt der Behinderung des Blatstromes mittels des Gefäßknotens wird die Gefäßerweiterung durch eine schwere Wanderkrankung erklärt. Die Befunde von Sachs, Knape, Hirschberg und Wintersteiner sollen zeigen, daßähnliche Wandveränderungen bei Gliomen nicht zu den Seltenheiten gehören, daß im Tumorgebiete Varikositäten und Aneurysmen, in den verschonten Netzhautpartien schwere entzïndliche Wandveränderungen lokalisjert sein können. Das Wesentliche und Primäre sei die Gliawucherung, ebenso wie in den Gliomen des Zentralnervensystems, dagegen sei die Wuoherung der Gefäße eine sekundäre im Sinne von Borst, womit auch die Möglichkeit gegeben wäre, daß letztere gelegentlich fehlen könne. Das Wachstum ist nach seiner Auffassung infiltrativ, die Netzhaut wird nicht in eine knotige Geschwulst umgewandelt, sondern ziemlich gleichmäßig verdickt. Meller schlägt den Namen Gliosis retinae diffusa teleangiectodes vor und führt noch an, daß gegen die Angiomnatur dieser Bildung auch dieser Umstand spricht, daß, wenn die Gefäßknäuel primür wären, so müßten Fälle bekannt sein, in denen ausschließlich eine Angiombildung vorhanden wäre. Weiter müßten bei reinen Angiomen Blutungen eine größere Rolle spielen, jedoch haben hier sichtbare Blutungen nur eine untergeordnete Rolle. Erinnert auch an das Verhalten der Aderhautangiome, welche keine reaktiven Veränderungen im Gewebe erzeugen, das umliegende Gewebe atrophiert nur in diesen Fällen. Erwähnt sei noch, daß Goldzieher ${ }^{2}$ ) bereits früher rein klinisch zum selben Resultat gelangte, gestützt auf die Überlegung, daß nicht nur Lichtsinn, sondern, trotzdem daß der Prozeß so hochgradig vorgeschritten war, daß die vorgetriebene Netzhaut die hintere Linsenfläche erreichte, noch gute Sehschärfe bestand. Aus diesen Symptomen folgerte er, daß die ungemein empfindliche Stäbchen- und Zapfensehicht nicht angegriffen sein könne, daß ein hyperplastischer Prozeß in den Innenschichten der Netzhaut vorliegen muß und belegte es mit dem Namen Degeneratio fibromatosa interstitialis retinae.

Es möchte zu weit führen, auch die wichtigen Gegenargumente v. Hip pels anzuführen. Es wurde schon bereits oben angeführt, daß die Auffassung des Prozesses als eine Angiomatose breiten Anklang fand und daß auch Leber diese anerkannte. Es schien uns nur darum notwendig, dieses Bild und die zwei entgegengesetzten Entstehungsmöglichkeiten anzuführen, nachdem in jüngster Zeit auch Meinungen auftauchten, wonach die strenge Isolierung dieser Erkrankung von der Retinitis exsudativa externa nicht beibehalten wird. - So beobachtete Gourfein. Welt ${ }^{3}$ ) bei einem 16 jähr., hereditär nicht belasteten jungen Manne am rechten

1) Meller, Utber das Wesen der sog. Hip pelschen Netzhauterkrankung. Dieses Arehiv, 85, 255. 1913.

2) Goldzieher, Über einen bisher noch nicht bekannten Augenspiegelbefund. Zentralbl. f. prakt. Augenheilk. 23, 65. 1899.

3) Gourfein - Welt, In weloher Beziehung steht die Retinitis exsudativa zu der Angiomatose der Netzhaut? Klin. Monatsbl. f. Augenheilk. 65, 705. 1920. 
Auge ein ganz kleines Angiom der Maculagegend, am linken Auge war temporal von der Papille ein großer weißer Herd, durch eine Arterie geteilt. Die obere Hälfte hatte das Aussehen einer verdickten Membran, die untere Hälfte hatte flottierendes Aussehen. Die Arterie wurde der Peripherie zu breiter und geschlängelt. Nach Gourfein - Welt handelte es sich im linken Auge um ein Spätstadium der Angiomatose, in welchem die Unterscheidung von Retinitis exsudativa nicht möglich gewesen wäre, wenn nicht das kleine Angiom des rechten Anges auf eine doppelseitige Angiomatosis hingewiesen hätte, Nach Verf. ist die Coatssche Retinitis in den meisten Fällen nur so ein Spätstadium der Angiomatose, deren Beginn unbeobachtet blieb. Gleichfalls könnten die Leberschen Netzhautdegenerationen durch multiple Aneurysmen und auch gewisse Blutungen der äußeren Schichten das Bild der Retinitis exsudativa verursachen. Letztere sei keine einheitliche Erkrankung, gleiche der Retinitis proliferens. Ähnlich wie letztere eine bequeme Bezeichnung für ein besonderes Bild der Retinalveränderungen nach Blutungen den inneren Netzhautschichten darstellt, so umfaßt die Retinitis exsudativa Veränderungen und Blutungen in den äußeren Netzhautschichten. Speziell die Coatssche Erkrankung entspräche nur den Endstadien der Angiomatosis retinae, wo eben das Angiom Blutungen in den Außenschichten verursachte. - Erggelet ${ }^{1}$ ) beschreibt auch einen Fall, in welchem in Gegensatz zum klinischen Befund zu der Angiomatosis retinae anatomisch das Bild der Retinitis exsudativa externa deutlich im Vordergrunde stand. In diesem Falle handelte es sich um ein 15 jähr. Mädchen; am linken Auge war klinisch das Vollbild der Angiomatose zu verzeichnen, ein großer vorspringender roter Knoten, zu dem zwei peripherwärts sich gewaltig erweiternde und geschlängelte Gefäße hinzogen. In der Macula eine Sternfigur, im übrigen Hintergrund kleine weiße Flecken und Flächen. An dem schon früher total erblindeten rechten Auge vollständige Netzhautabhebung, dunkelrote, zum Teil geschlängelte und an einer Stelle durch Kaliberschwankungen auffallende Gefäße in der grauen Netzhaut. Am Ende eines dicken geschlängelten Gefäßes war ein hellrotes Knötchen, welches bei 35 facher Vergrößerung an einen Nierenglomerulus erimnerte. Mikroskopiseh zeigte sich die Netzhant teilweise durch Exsudat abgehoben, schwere Veränderungen der Netzhaut, welche durchweg von außen her unterging; stellenweise ist es zum völligen Schwund der Netzhautelemente gekommen. Stäbchen und Zapfen fehlten, zum Teil außerordentlich erweiterte Gefäße, an einer Stelle auch in den Außenschichten. Vereinzelte knotenartige Verdickungen der abgelösten Netzhaıt. An dem klinisch beobachteten Knoten wurde kein Gefäßreichtum gefunden, in der Netzhaut ausgesprochene Gliawucherung, und an der nicht abgelösten Seite waren Retina und Chorioidea durch eine von der Aderhaut dureh das Pigmentepithel nicht überall alogegrenzte Gewebsmasse verbunden. Die Aderhaut becaß nur hier und dort kleine Rundzelleninfiltration.

Die Frage der Zugehörigkeit beider Fälle soll vorläufig offenbleiben. Jedenfalls zeigen diese, daß beide Krankheitsgruppen noch nicht genau umschrieben und umzingelt sind, und evtl. auch Grenzfälle vorkommen können, wo eine genaue Unterscheidung auf Schwierigkeiten stoßen kann. Wir werden ja weiter unten sehen, daß auch innerhalb der Gruppe der Retinitis exsudativa externa ähnliche Unsicherheiten bezüglich der Symptome, der Genese und des Wesens gleichfalls Hand in Hand gehen. Dieser Umstand beruht vielleicht darauf, daß äußerst selten 1920.

1) Erggelet, Angiomatosis retinae. Klin. Monatsbl. f. Augenheilk. 65, 413. 
Frühstadien untersucht werden konnten, und wir glauben, daß in dieser Beziehung unser Fall wohl als alleinstehend betrachtet werden muB und so Interesse beanspruchen kann.

\section{Krankengeschichte.}

G. M., $1^{1} / 2$ Jahre altes Mädchen, aufgenommen am 19. VII. 1920.

Das Kind wurde Anfang Mai von den Eltern wegen beginnendem Strabismus zu dem Augenarzte Dr. Schür ma n n gebracht. Dieser konstatierte im linken Auge am Sehnervenkopfe eine beginnende, auf Gliom verdächtige Geschwulstbildung. Das Kind blieb in Kontrolle bis Mitte Juli; da ein Fortschreiten zu konstatieren war, wünschten die Eltern anch Professor Sidler zu konsultieren. Professor Sidler nahm das Kind zur weiteren Beobachtung in die Klinik auf.

Status praesens: Gesund aussehendes, sehr kräftiges, aufgewecktes Kind. Vater und Mutter vollständig gesund; es ist das erste Kind in der Ehe. In der Familienanamnese keine Anhaltspunkte für Lues oder Tuberkulose. In Aszendenz angeblich kein Gliom.

Rechtes Auge reizlos, Cornea klar. Vorderer Bulbusabschnitt ohne Besonderheiten, ophthalmoskopisch normale Verhältnisse. Sehschärfe nicht zu bestimmen, hingegen ist sicher, daß das Kind mit diesem Ange gut sieht.

Linkes Auge gleichfalls reizlos, steht in leichter Konvergenzstellung. Bulbusbewegungen scheinen nach allen Seiten frei zu sein. Vorderer Bulbusabschnitt ohne Besonderheiten. Das Kind scheint mit diesem Auge fast nichts mehr zu sehen, wird sehr böse und weint, wenn man das rechte, gesunde Auge zubindet.

Fundus: An Stelle des Opticus eine markig-weiße Masse, Gefäßtrichter noch gut zu erkennen (Abb. 1 zeigt genau die Verhältnisse bei der Aufnahme). Rings um den Opticus in der Ausdehnung von drei Papillenbreiten ein flachhöckeriger Tumor, von bläulichgelber bis bräunlicher Farbe. Früher war die Farbe noch weiß. Abhebung der Netzhaut oder Blutungen nirgends zu sehen. Gefäßveränderungen nicht vorhanden. Peripherie überall normal.

Nachdem der Tumor allmählich zunimmt, wird das Auge am 1. VIIT. 1920 in Äthernarkose enucleiert.

Normaler Heilungsverlauf.

Nachdem das Ange. wegen Gliomverdacht enucleiert wurde, wird die linke Orbita am 10. VIII. vor der Entlassung aus der Klinik röntgenbestrahlt.

Das enuoleierte Auge wurde lebenswarm in Formalin gelegt.

Pathologisch - a natomischer Teil: In dem nach Formalin- und Alkoholhärtung äquatoriell aufgeschnittenen Bulbus war die Netzhaut überall anliegend. In der Gegend der Opticusscheibe zeigte sich eine weißlich-graue Verdickung, über welche Retinalgefäße zogen. Der hintere Abschnitt wurde in Paraffin eingebettet und in Serien zerlegt. Die Schnitte wurden mit Hämatoxylin, Hämatoxylin-Eosin, Delafield, Mallory, weiter nach Weigerts Fibrin- und Elastikafärbung gefärbt.

Die mikroskopische Untersuchung ergibt, daß die pathologischen Veränderungen die Papille und die der Papille ringförmig angrenzenden Netzhautteile betreffen; in einer Ausdehnung von ungefähr 2,5-3,0 mm. Das Aussehen der veränderten Partien ist recht variabel, und in den verschiedenen Schnitthöhen ergeben sich voneinander abweichende Bilder.

Allen Schnitten ist gemeinsam, daß die hochgradigen Veränderungen a $m$ hinteren Pol lokalisiert sind. Zwischen Netzhant und Aderhaut ist überall ein schmaler Spalt vorhanden, die Netzhaut liegt nirgends der Aderhaut an, doch ist diese gleichmäßige Abhebung eine artifizielle, im subretinalen Raum sind weder Blutungen noch Exsudat vorhanden. Die Netzhaut ist nur an den erwähnten 
Stellen breiter geworden, die peripherischen Netzhautteile sind nur etwas breiter als normal und dies wird auch hauptsächlich durch Alteration aller inneren Schichten bedingt. In den angrenzenden Glaskörperpartien ist eine der Netzhaut anlifgende Auflagerung, die später noch genauere Besprechung findet. Ringförmig um der Papille ist die knospenartige, bucklige Vortreibung der Netzhaut, welche in allen Richtungen die schon erwähnte gleichmäßige Auftreibung besitzt. Die vorspringende Partse geht ziemlieh steif mit abfallenden Rändern in die verhältnismäßig normale Netzhaut über. Die Betrachtung mit keinen Systemen zeigt deut. lich, daß sämtliche Schichten der Netzhaut innerhalb dieses Bezirkes in toto ge*

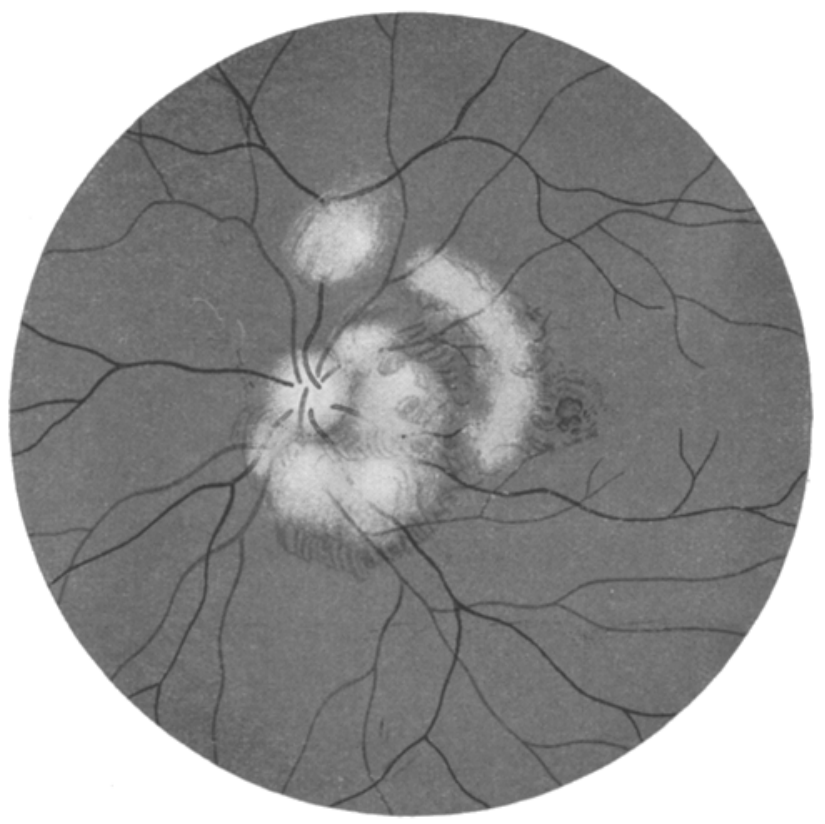

Abb. 1. Bei der Anfertigung des Bildes waren noch drei isolierte Herde vorhanden. Im späteren Verlaufe sind diese versohmolzen, und dadurch entstand rings um den Opticus ein flachhöckeriger Tumor. Die Gefäße waren teilweise sichtbar, teilweise gedeckt.

faltet sind. Zwischen Fältelung der äußeren und inneren Netzhautschichten besteht eine Disharmonie. Die oberhalb und unterhalb der Papille gelegenen Schnitte zeigen ausgesprochener die Art der Fältelung. Diese besitzen in den inneren Schichten 6-8 Einbuckelungen von verschiedener Tiefe, deren Konvexität der Aderhaut zugewendet ist. In den mittleren Teilen der Vortreibung sind die einzelnen Einbuchtungen breiter und tiefer, in den abfallenden Randteilen schmäler und seichter. Letzteres Verhalten wird besonders schön durch die innere plexiforme Sohicht markiert, welche eine gleichmäBig breite, wellenförmige, einer Sinuslinie gleichende Konfiguration besitzt und zwischen den verdrängten Körnersohichten genau die topographische Lage anzeigt.

Gegen diese mäßige Fältelung der Netzhautinnenschichten zeigen die äußeren Schichten der Netzhaut eine vielfache Fältelung. In den Außenschichten läßt sich die Zahl der einzelnen Falten nicht einmal annähernd mehr bestimmen. Schichtenweise und auch stellenweise im selben Schnitt entstanden dadurch recht 
verschiedenartige Bilder. In den Randpartien, temporal und nasal gleichfalls, befinden sich noch Einbuchtungen verschiedener Tiefe, in welchen die normale Schichtung noch deutlich zum Vorschein kommt und deren Konvexität dem Glaskörper zugewendet ist $(A b b .2$, mit kleiner VergröBerung gezeichnet, veranschaulicht ganz naturgetreu diese verschiedenartige Fältelung der äußeren und inneren Netzhautschichten). Oft findet man schlauch-oder ringförmige Konfigurationen infolge Abkappung der Falten durch die Schnittführung. Diese Gebilde lassen meistens nooh Reste der Stäbohen- und Zapfenschicht erkennen, die Membrana limitans externa markiert sich noch als scharf konturierte Linie, darunter verschiedene Mengen Körner der äuBeren Körnerschicht. Die Verfolgung der Serien zeigt

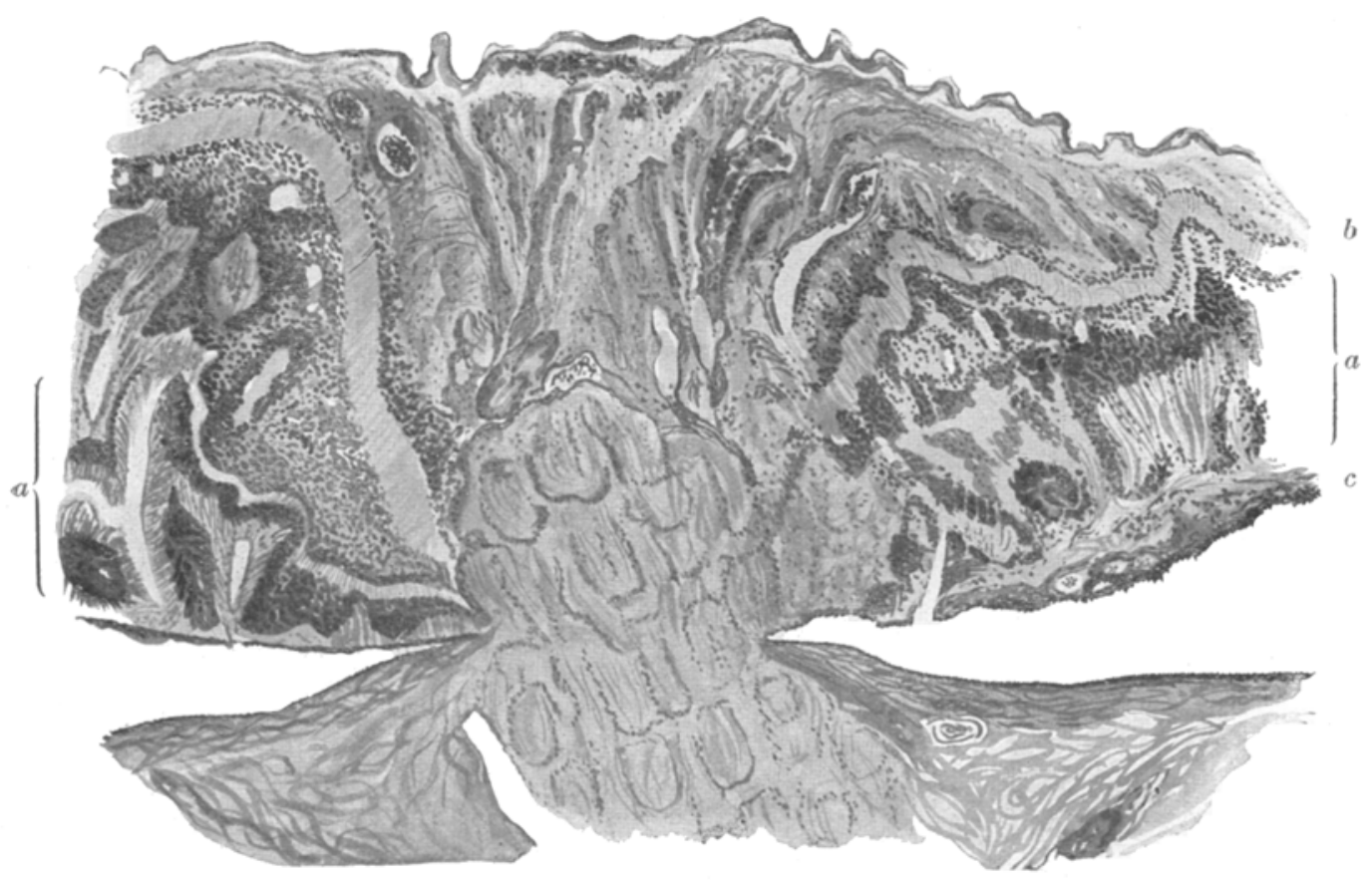

Abb. 2. $a=$ starke Fältelung der Außenschichten mit schlauch- und ringförmïgen Figuren; $b=$ Vorbuckelungen der inneren Schichten; $c=$ eingesohobene Zwischensehwarte mit jungen BlutgefäBen und mit reichlicher Pigmentbelastung.

es, daß für die Menge der Körner die Verdrängung durch Faltenbildung verantwortich ist. Oft finden sich Stellen, in welchen die versehiedenen Falten das Bild dermaßen komplizieren, daß die Übersicht verloren ginge, wenn eben die Serienschnitte nicht die reishliche Fältelung erweisen möchten. Unter und neben den schlawch- und drüsenartigen Einstülpungen kömen Quer, Längs* oder Schrägschnitte derselben Falten sich befinden. In dem rasch wechselnden Aussehen tauchen stellenweise Insel- und Rosettenformen auf. Einige ähneln sehr den Winter . steiner schen Rocetten, mit welchen sie aber selbstredend nichts zu tun haben. Nur die ringförmige Anordnung der Körner, die deutliche Membrana limitans exterra und das Lumen dieser Bildungen zwingt zum Vergleiche mit den gleichfalls ringförmig angeordneten, mit scharfer Zellmembran versehenen Neuroepithelien der 
Netzhautgliome. Auch kann es gelegentlich vorkommen, daß in den zentralen Lichtungen unserer Gebilde einzelne Zellen sich vorfinden.

Nach diesem allgemeinen Utberblick können wir uns den Veränderungen der einzelnen Schichten zuwenden. Um eine bessere Übersicht zu ermöglichen, werden die Veränderungen der stark veränderten zentralen (in der Umgebung des binteren Poles) und der kaum veränderten peripheren Netzhautteile voneinander $a b$ gesondert betrachtet.

Am hinteren Pol, wo die Fältelung so stark ausgesprochen ist, findet man dadurch keine zusammenhängende Linie der Stäbchen-und Zapfenschicht. Die Schicht der Stäbohen und Zapfon ist aber äberall erhalten, zum größten Teil aber stark verändert, indem Stäbchen und Zapfen blasig aufgetrieben sind, so daß an manchen Stellen die typische Zeichnung verlorengegangen ist. Die Membrana limitans externa ist in Form einer scharfen Linie abgebildet. Die beiden Körnerschich ten und die äußere retikulare Schicht sind im ganzen wohlerhalten; sie beteiligen sich an den beschriebenen Windungen, ihre Elemente sind aber gut erhalten geblieben. In diesem Gewebe fallen kleine cystoide Bildungen auf, welohe nur hier und da vorkommen, rundlich, leer und von verschiedener GröBe sind. Die Lücken sind im ganzen als kleine zu bezeichnen, die größeren sind teilweise von ovaler Form und scharfer Begrenzung, ihre Größe schwankt zwischen 2-6facher der Größe der Zellen der Körnerschicht. Außerdem finden wir besonders in den äußeren Schichten an ganz vereinzelten Stellen kleinste fibrinöse, nach van Gicson rötlich gefärbte, völlig strukturlose, geronnene, intra vitam wahrscheinlich flüssig gewesene, eiweiBreiche Massen eingelagert. Ähnliche fibrinöse Exsudation ist auch an der AuBenfläche der Retina vorzufinden, zwischen den drüsenartigen EinstüJpungen der AuBerfläche, wodurch die schmalen und tiefen Finsenkungen ausgefüllt oder verklebt erscheinen können. - Von degenerativen Veränderungen der Zellen in diesen Schichten kann nach sorgfältigster Untersuchung auch nicht gesprochen werden. - In der inneren retikulären und der Faserschicht ist keine nennenswerte Alteration zu finden außer stärkerer Entwicklung faseriger Züge, welche auf Gliawucherung zurückgeführt werden können. In der Schicht der Ganglienzellen befinden sich aber schon bemerkenswerte Veränderungen (die Veränderungen der Gefäße werden später für sich behandelt), nämlich Degeneration der Ganglienzellen and Höhlenbildung in ihrer Umgebung, welch letztere auch einige rötlich tingierte amorphe Körnchen aufweisen können. Die Ganglienzellen erscheinen viel größer als normal, der Protoplasmaleib ist mächtig angeschwollen und schließt feine Körnchen ein, wodurch ein granuläres Aussehen hervorgerufen wird. Der Kern liegt meistens exzentrisch in einem Pol der Zelle gelagert, oft auch in seiner Gestalt deutlich verkleinert, und stellenweise pyknotisch. Neben diesen degenerierten Ganglienzellen befindet sich eine größère Anzahl kleiner, stark gefärbter Kerne, welche besonders neben und in der Papille an Zahl stark zunehmen. Ein Teil dieser Zellen entstammt der Gliawuoherung; diese sind rundlich, manchmal vergrößert, mit kaum sichtbarem Protoplasmaleib, stellenweise mit Vakuolen. Außer den Abkömmlingen der Glia sind auch Zellen anderer Herkunft vorhanden, welche sehr Endothelzellen ähneln. Die Kerne sind̈ relativ groß, länglich oder rundlich, spärliches, in Körnchen angeordnetes Chromatin enthaltend, mit ein bis zwei Kernkörperchen, und infolge des spärlichen Chromatingehaltes weniger intensiv gefärbt als die Gliazellen. AuBerdem sind Zellen vorhanden, bei denen die Hingehörigkeit genau zu bestimmen nicht möglich ist; diese kömnten nach manchèn Eigenschaften des Aussehens bald der Glia, bald den endothelahnlichen Zellen zugesprochen werden. - In der Ganglienzellenschicht sind die zuletzt erwähriten Zellformen nur spärlich anwesend, dagegen nehmen diesë an Zahl im stark ödematösen Papillengewebe rapid zu, worauf wir noch zurückkommen. - Die Me mbra na 
limitans interna ist an einigen Stellen durch amorphe Exsudatkörnchen abgehoben, und so sind die Müllerschen Stützfasern schön verfolgbar. Erwähnt sej. noch, daß an 2-3 Stellen zwischen der Limitans interna und den Ganglienzellen ganz kleine Blutunge $n$, bestehend aus 5-10 roten Blutkörperchen, eingebettet sind. Diese sind aber die einzigen Stellen in der Netzhaut, wo solche überhaupt auffindbar gewesen, ganz sicher fehlten diese vollständig in den äuBeren und mittleren Netzhautschichten. Auf die Limitans folgt nach innen eine zarte Schicht feinfaserigen Gewebes, welches aus Lamellen besteht, die paralle 1

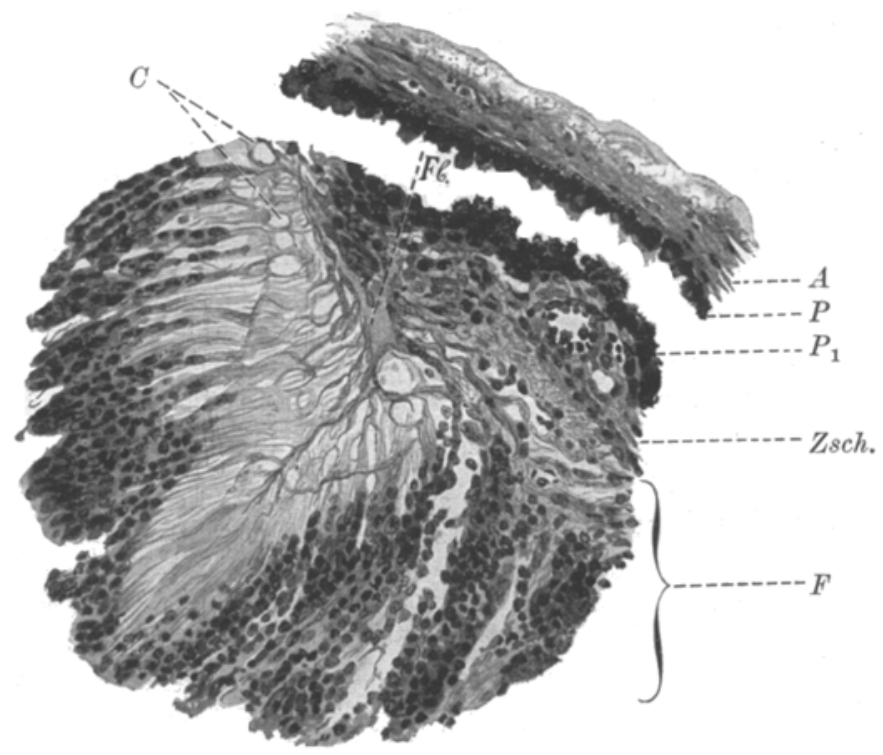

$\mathrm{Abb}, \mathrm{B}, A=$ Aderhaut $; P=$ Pigmentepithelschicht; $Z$ sch $=$ eingeschobene $Z$ wischenschicht; $P_{1}=$ Pigmentiberzug der $\mathrm{Zwischenschicht;} F b=$ Fibrinablagerung an der Außenfläche der Net $z-$ hant; $C=$ kleine Hohräume; $F=$ Falten der AuBenschichten der Netzhaut.

miteinander und mit der Innenfläche angeordnet sind. Zwischen diesen lamellären Auflagerungen sind rote Blutkörperchen zerstreut. Stellenweise sind längere Strecken zwischen den Lamellen mit letzteren gefüllt, meistens befinden sich aber nur 2-3 Lagen roter Blutkörperchen übereinander.

Veränderungen des hinteren Poles wie die Veränderungen der Gefäße, der Papille and die der zwischen Netzhaut- und Aderhaut eingelagerten Zwischenschicht müssen abgesondert betrachtet werden.

Bei der Beschreibung der einzelnen Schichten wurde schon Erwähnung getan, daß die Einsenkungen der Falten mit rötlichem Exsudat ausgefüllt waren, die scheinbar mechanisch die Fältelung verursachten. An der Oberfläche der Netzhaut läßt sich ein ähnliches Exsudat nirgends nachweisen. Dagegen befindet sich in dem stark veränderten Gebiete $z$ wischen Netzhaut und Aderhaut eine förmliche $Z$ wischenschicht eingelagert, welche in der Peripherie zugespitzt ist und entsprechend den kaum veränderten Partien sofort aufhört. Bei der Präparierung des Bulbus ist es zu einer Ablösung der Netzhant gekommen, diese $Z$ wi sehenschicht haftet aber in sämtlichen Schnitten der Netzhaut an. Dadurch ist der subretinale Raum beiderseits durch Pigmentbelag gekennzeichnet. Die Ver- 
bindung zwischen Netzhautoberfläche und Zwischenschicht ist eine feste und lückenlose, stellenweise besitzt diese eingelagerte Schicht auch kleine Sprossen, welche in die schlauchartigen Einsenkungen der Netzhautfalten hineinragen. Die Zwisohenschicht ist ungefähr gleichmäßig gebildet, ihre Dicke entspricht ungefähr der Hälfte der Breite der normalen Netzhaut. Die Grenzen zwischen ihr und der Netzhaut sind überall gut zu erkennen. In den der Aderhaut zugewendeten Teilen befindet sich eine starke Pigmentierung. An der Oberfläche der eingelagerten Sehicht ist in Form einer zusammenhängenden Linie eine stark pigmentierte Schicht, deren Breite leichten Schwahkungen unterworfen ist. Die Pigmentschicht besitzt nach außen zu kleine Vorbuckelungen, zwischen denen dünnere Lagen sich befinden. Hier sind die Zellen dermaßen mit Pigment beladen, daß die Zellform der pigmentbeladenen Zellen völlig gedeckt ist. Die darunterliegende faserarme, zellreiche Schicht hat besonders in den oberflächlichen Lagen gleichfalls viel Pigment und auch vereinzelte Fuscinkörner. Hier bildet die Pigmentierung schlauch- und brückenartige Figuren, besonders ist es auffallend, daß einzelne Gefäße vom Pigment umringt werden (Abb. 4 zeigt so ein in Pigment eingebettetes Gefäß, auch in der weiteren Umgebung ist die beschriebene Art der Pigmentierung ersichtlich). Den tieferen Lagen geht das Pigment ab, nur vereinzelte Pigmentkörner sind, eventuell freiliegend, nachweisbar. - Die Zwischenschicht wird aus dichtgelagerten Kernen gebildet. Die Kerne besitzen meistens spindelige, längliche

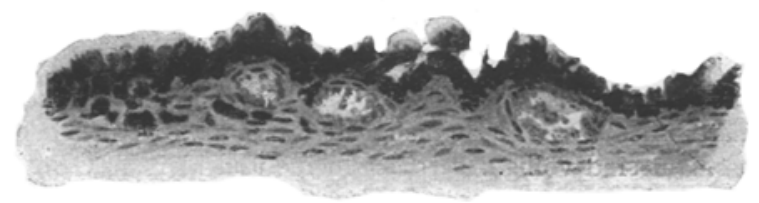

Abb. 4. Ehe Stelle der Abbildung 3 mit starker Vergröernng betrachteb. Unehenheiten im Pigmentüberzage, große Blutgefaße mit dunner Wandung, Finwanderung und Ablagerung des Pigmentes in der Umgebung der Gefäße.

ausgezogene Form, die rundlichen sind viel seltener. Die Längsachse der Kerne ist parallel der Oberfläche gelagert, und dadurch heben sich diese von der darunter. liegenden Netzhaut immer scharf ab. Die Kerne sind intensiv gefärbt mit Kernfarb. stoffen. Vereinzelt sind auch Lymphocyten und mehrkernige Leukocyten zerstreut, Plasmazellen, Mastzellen und eosinophile Zellen waren nicht auffindbar. Die Schicht führt auch eine beträchtliche AnzahI neugebildeter Blutgefäße, darunter einige ziemlich große, beinahe die ganze Dicke der Schicht einnehmende, mit roten Blutkörperchen ausgefüllte. - Intra vitam mußte diese Schicht der Aderhant genau anliegend gewesen sein. Diese Auffassung ergibt sich daraus, daß in diesem Gebiete auch die Pigmentepithelien der Aderhaut Veränderungen aufweisen und daß im subretinalen Raum nirgends Exsudat nachweisbar war.

Die Papille ist stark geschwollen und ödematös. Die Fasern weichen stark auseinander. Bereits bei der Beschreibung der Zellen der Ganglienzellenschicht der Netzhaut wurde Erwähnung getan, daB in dieser Sohicht viele kleine Zellen, Derivate der Glia- bzw. Endothelzellen, nachweisbar gewesen sind, und daB die Zahl letzterer im Gewebe der Papille rapid zugenommen hat. Besonders stark wird die gliöse Entartung der Papille in dem über dem Gefäßtrichter liegenden Teil (s. Abb. 5), wo das Gliagewebe feine, leere Maschenräume einschließt und wo eine erhebliche Faservermehrung stattgefunden hat. Diese Partie mit den vermehrten Fasern hebt sich von der Ungebung ziemlich scharf ab. In diesen Teilen werden die Zellen, deren Herkunft nicht sicher zu entscheiden war (Glia- bzw. Endothelkerne), ziemlich häufig. Das sind die größeren Zellelemente mit rundlichem oder ovalem 
Kern und mäßigem Chromatingehalt. Die sichere Entscheidung kann nicht getroffen werden, doch ist es bemerkenswert, daß eben an dieser Stelle sehr zahlreich ganz kleine ne ugebildete Blutgefäßehen von der Größe der Präeapillaren zusammengehäuft sind und ein Teil derselben den später zu beschreibenden Endothelwucherungen nnterworfen ist. Somit wäre die Annahme, daß diese Zellen endothelialer Herkunft sind, doch naheliegend.

Schließlich sollen die Veränderungen der Gefäße behandelt werden. In den inneren Netzhautschichten wurden viele große Gefäße gefunden, in den zentralen Teilen der Papille zahlreiche kleinere und gleichfalls viele neugebildete Gefäße in der Schwarte, letztere bedeutenderen Kalibers. In der Ganglienzellenschicht, besonders in der Umgebung der Papille, liegen 2-3-4 Gefäßquerschnitte ziemlich oft nebeneinander. Die Gefäße sind meistens weit, klaffend, alle mehr oder weniger pathologisch umgestaltet. Einige enthalten zirkulierendes Blut, andere sind direkt mit roten Blutkörperchen zum Prallen gefüllt. Leukocyten sind nur hier und da zu treffen, ausgesprochene Randstellung der weißen Blatkörperchen ist selten. Hyalinisierung der Gefäßwände war oft nachweisbar. Sehr häufig zeigten die Endothelien pathologische Veränderungen. Die Endothelien waren vielfach gequollen und gewuchert. Die Wucherung erreichte verschiedene Grade. In einigen Gefäßen ist es nur zur Verunstaltung des Lumens an einer Seite gekommen, in das Lumen springt eine aus Endothelzellen gebildete Halbinsel hinein, in anderen GefäBen ist es durch konzentrische Wucherung zu einer mehr gleichmäßigen Verengung der Lichtung gekommen. Abb. 6 stammt aus den inneren Schichten und zeigt eine geringgradige Entwicklung der Endothelwucherung. An zahlreichen Stellen führte aber diese Wucherung zu einem vollständigen Verschluß des Gefäßlumens. Hauptsächlioh an der Papille und in der Ganglien- und Opticusfasernschicht sind völlig verstopfte kleine und kleinste Gefäße eingebettet, wo nur eine Anhäufung von Endothelzellen sichtbar ist, neben Gefäßen, welche durch die starken Endothelveränderungen noch eben ein kümmerliches Lumen aufweisen. Es gehört nicht zu den Seltenheiten, daß solche proliferierende Epithelzellhaufen in das Gliagewebe eingelagert sind, und daß es nur vermutet werden Jann, daß diese früher Gefäße darstellten. - Die zweite Gruppe der Gefäßveränderungen betrifft die Adventitia. Neben den Endothelwucherungen sind hochgradige Wucherungen im Adventitialmantel vorhanden, und dadurch erhalten einige Gefäße ganz breite Ringe. Zwischen der gewucherten Adventitia und dem Gliagewebe ist meistens ein schmaler, leerer Spalt vorhanden, und so wird die Abgrenzung vom Grundgewebe eine deutliche. - Die Adventitialwucherungen sind nicht nur auf die abgehobene und vielfach gefaitete Netzhaut beschränkt, auch deren abwärtsliegende Teile sind dadurch getroffen, insbesondere die Gefäße der Papille vor der Lamina cribrosa. In dem verschieden breiten Adventitialring sind stellenweise die einzelnen Zellen gut zu erkennen, sie scheinen etwas dunkler tingiert zu sein als die proliferierenden Zellen des Lumens. Beim Durehmustern vieler Präparate können Momente auftauchen, daß es nicht sicher zu entscheiden wäre, ob diese Zellen Abkömmlinge der Endothelien oder der Adventitialzellen darstellen, keinesfalls läßt sich aber ein Zusammenhang mit gliös gewucherten Zellen erkennen.

Die Veränderungen der außerhalb dieses Bezirkes liegenden Netzhautpartien, welche kurzweg periphere Netzhautteile genannt werden mögen, lassen sich kurz zusammenfassen. -

Die Membrana limitans interna zeigt mit Unterbrechungen Abhebungen bis zu der Ora serrata. In der Schicht der Ganglienzellen sind ähnliche Veränderungen geringeren Grades als in den zentralen Teilen. Die Ganglienzellen sind hier gleichfalls gequollen, der Kern peripheriseh gelagert. Anch hier ist es zur Höhlenbildung 
gekommen, in welchen amorphe Exsudatkörner zu sehen sind. Die fëine fibrilläre Auflagerung im anstobenden Glaskörper ist ähnlicherweise aufgebaut als in den stark veränderten Teilen. Bemerkenswert ist das Verhalten des Stratum plexiforme externum, welches ausschließlich aus kleinsten Hohlräumen besteht. Die Hohlräume sind durchweg leer, die Abgrenzung wird von 1-2 ausgezogenen Fasern gebildet. Blutungen enthalten diese Hohlräumchen nirgends. Im ganzen wirken die Hohlräume, als wenn eine mechanische Auseinanderziehung bzw. Spaltung der Netzhaut vorliege (ähnlich wie bei Tumoren der Aderhaut manchmal beobachtet wird), besonders weil diese Veränderung neben den stark vorgebuckelten Retinateilen sofort sichtbar wird und bis zu der Ora serrata noch an Ansdehnung allmählich zunimmt. Die Körnerschichten, weiter die Schicht der Stäbchen und Zapfen ist an der Peripherie überall gut erhalten. - Die Aderha ut ist kaum ver. ändert. Der Gefäßgehalt kann als vermindert bezeichnet werden. Die Vitrea ist überall vorhanden. Zerstreut findet man kleinzellige Infiltration. Diese Zellen sind aber zerstreut, ballen sich nirgends zu Herden zusammen. Die Pigmentepithelien zeigen überall das gewohnte Bild, mit Ausnahme der Stellen, wo die eingeschobene Schwarte gelagert ist. An letzterer Stelle hat die Schicht eine höckerige Oberfläche, Stellen, wo eine ausgesprochene Wucherung vorhanden ist, wechseln mit anderen $a b$, wo die Zellen ihr Pigmentgehalt teilweise eingebüßt haben. Gefäßübertritt aus der Aderhaut in die Schwarte und ebenso zellige Emigration aus der Aderhaut wurden nirgerds angetroffen.

Epikrise. Bei einem 11/2 jährigen Mädchen war Strabismus converge ns des linken Auges aufgetreten. Durch diese Stellungsanomalie wurden die Eltern veranlaßt, das Kind fachärztlich untersuchen zu lassen. Diese Untersuchung ergab es, daß a m hinteren Pol des betreffenden Auges, in der Umgebung der Papille eine weißliche, von Retinalgefäßen überzogene Masse in die Netzhaut eingelagert gewesen ist. Entzündliche Erscheinungen, Blutungen waren nirgends vorhanden. Es wurde an Gliom gedacht. In der späteren Beobachtungszeit hat diese Masse an Ausdehnung rasch zugenommem, und so wurde die Entfernung des Auges beschlossen. Erst die nachfolgende pathologisch-anatomische Untersuchung vermochte das Bild zu klären. Es sei noch erwähnt, daß während der Beobachtungszeit, als das tumorartige Wachstum rasche Fortschritte machte, auch jegliche klinische Entzündungserscheinungen oder Blutungen vermißt wurden. Veränderungen traten nur insofern auf, als die weißliche Farbe der in den tiefen Netzhautschichten liegenden Masse an der Peripherie eine leichte schiefergraue Farbe bekommen hat. Gröbere Gefäßwandveränderungen konnten klinisch nicht aufgefunden werden.

Erst der erhobene anatomische Befund zeigte, daß wir mit Veränderungen zu tun hatten, welche höchstwahrscheinlich in diese Gruppe der Erkrankungen gehören, die schon auch früher gelegentlich beschrieben wurden und unter verschiedenen Namen in der älteren Literatur auffindbar sind. Coats' Verdienst bleibt es aber, diese in ihrer Deutung diskrepanten Befunde in eine einheitliche Gruppe geeinigt zu haben, unter dem Namen Retinalerkrankung mit massiger 
Exsudation oder Retinitis exsudativa. Sicher ist die Zahl der in diese Gruppe gehörenden Fälle bedeutend größer, als es allgemein angenommen wird. Besonders wird die Zahl der Pseudogliome stark zurückgehen, wenn die Pseudogliomfälle anatomisch untersucht wurden unter Berücksichtigung der Veränderungen dieser Gruppe. Ich selbst habe Gelegenheit gehabt', 16 Fälle von Gliomen ${ }^{1}$ ) anatomisch zu untersuchen, und habe drei Fälle gefunden, welche sicherlich in diese $A b$ teilung gehören. Bei unserer Besprechung werden wir aber diese nicht berücksichtigen, nachdem diese Spätstadien der Erkrankung in allen Fällen mit totaler trichterförmiger Netzhautabhebung darstellten und somit in der Beurteilung der Tnitialveränderungen nicht verwertet werden können.-

Coats ${ }^{2}$ hat im Jahre 1908 und 1912 in seinen Arbeiten die obige Krankheitsform zusammengefaßt, wo er sich auf 10 eigene, davon 9 pathologisch-anatomisch untersuchte Fälle gestützt hat. Er rechnet in seiner Arbeit viele Fälle der Literatur, welche eigentümliche Veränderungen der Netzhaut oder atypische Retinitis circinata, weiter Tbe. der Netzhaut oder Chorioiditis exsudativa von den Autoren benannt wurden, dieser Krankheitsform zu.

Das Wesentliche und Charakteristische dieser Krankheitsform sei die Einseitigkeit des Prozesses, die Anhäufung einer ausgedehnten, opaken, undurchsichtigen Exsudation oder Infiltration unterhalb der Netzhautgefäße, das Auftreten im jugendlichen Alter. Nach Leber blieb das Lebensalter der Betroffenen in $80 \%$ der Fälle unter 25 Jahren, nur in $20 \%$ war es höher. Der Verlauf ist langsam, schleichend, Coats beschreibt sogar einen Fall, welcher 16 Jahre lang beobachtet wurde. Im späteren Verlaufe kommt es meistens unter Hinzutritt von Netzhautablösung, Iridocyclitis, Katarakt, Glaukom usw. zur Erblindung und Verlust des Augapfels.

Die pathologischen Merkmale sind schon weniger einheitlich. Coats unterscheidet in seiner ersten Arbeit Fälle mit und ohne auffallende GefäBveränderungen und Fälle mit arterio-venöser Kommunikation. Letztere. Fälle stünden nahe der v. Hippelschen Angiomatosis retinae, wo neben der Angiomatosis ausgebreitete Exsudatmassen auftreten.

Die Prädilektionsstelle des in mehreren Herden auftretenden Exsudates ist der hintere Pol oder die Gegend der Macula. Die Herde können konfluieren. Blutungen sollen sehr häufig sein, und bei stärkeren Gefäßveränderungen sollen diese ständig nachweisbar gewesen sein. Konstante, aber unwesentliche Veränderung ist das Vorhandensein einer Bindegewebsschicht zwischen Aderhaut und Netzhaut. Diese Bindegewebsschicht repräsentiert sich im Frühstadium locker, zellreich, dicht. In der Bindegewebsschicht sind Hohlräume mit Blutkörperchen, Leukocyten und Fibrin ausgefüllt. Das Exsudat ist mit den äußeren Netzhautschichten verbunden, in den Frühstadien besteht keine Verbindung mit der Netzhaut. Nur später kommt es zu einer Infiltration dè Aderhaut und zu Verwachsungen mit der Netzhaut, wobei aber die Bruchsche Membran unversehrt bleibt. Die

3) 17 Gliomfälle verdanke ioh der Liebenswürdigkeit des Herrn Primarius Dr. O. Purtscher in Klagenfurt, der mich mit Material unterstütze. Es ist mir eine angenehme Pflichtauch andieser Stelle meinen ergebenen Dank aussprechen zu dürfen.

2) Coats, Ophthalm. Hosp. Rep., 1908 und Über Retinitisexsudation (Ret. haemorrh. ext.). Dieses Archiv 81, 275. 1912. 
Blutversorgung der Zwischenschicht ist in den Frühstadien eine retinale, erst nach Auftreten der Verwachsungen kann es durch Proliferation und Einwanderung der Pigmentepithelien zur Auskleidung des Raumes durch Pigmentschicht an beiden Seiten, zu epithelumsäumten Röhrchen und Einhüllung der Gefäße kommen. Die Gefäße der betroffenen Teile können verschiedenartige Veränderungen zeigen, wobei die Venen eher befallen sind als die Arterien, und die Hauptveränderungen betreffen immer die kleineren Äste über die zweite und dritte Teilung der Gefäße hinaus. Häufig kommt eine Dilatation obne Wandveränderung vor und so die Vortäuschung eines kavernösen Angioms, weiter Thrombose, Verdickung der fibrösen Wand, hyaline Degeneration und Perivasculitis in den Iymphbahnen. Gelegentlich hat Coats auch Endothelproliferation beobachtet (Fall 2 und 10).

Was die Bedeutung dieser Symptome betrifft, so wird das Ha u ptgewicht a uf die Blutungen verlegt. Die Blutungen entstehen in den Außenschichten der Netzhaut, welche dann häufig nach deren Außenfläche durchbrechen, einem Zerfallsproze $B$ unterliegen und schließlich durch langsame Organisation in eine narbige Gewebsmasse umgewandelt werden. Die fibrinöse Durchtränkung soll gleichfalls von den Blutungen herrühren, weil Zeichen einer ausgiebigen oder schweren Entzündung, welche die fibrinöse Durchtränkung bedingen könnte, fehlten. Weiter bezeugt die Gegenwart von Cholesterin ebenfalls vorausgegangene Blutungen. Das mikroskopische Fehlen der Blutungen wird auf Organisationserscheinungen zurückgeführt.

Leber ${ }^{1}$, der bei Beschreibung der Erkrankungen der Netzhaut diese Gruppe ausführlicher Betrachtung unterworfen hat, konnte den Behauptungen von Coats nicht in jeder Hinsicht beistimmen. Das klinische Aussehen, weiter die Verlegung der primären Veränderungen in die Netzhaut ist beiden Ansichten gemein. Nur s pielen bei Coats in der Entstehung der Erkrankung die Hau ptrolle die Blutungen, und die übrigen Veränderungen entstünden aus Blutungen durch Organisation. Dagegen weist Leber den Blutungen nur eine Nebenrolle zu. Die Blu. tungen wären nur Nebenerscheinungen, die Hauptveränderung sei eîn Entzün. dungs- oder Nekrotisierungs prozeB der Netzhaut mit serofibrinöser Exsudation und massenhafter fettiger Degeneration und Desquamation des Pigmentepithels. Dementsprechend sei der ganze Vorgang nicht eine Reti nitis h a e morrhagica externa (Coats), sondern eine Retinitis exsudativa serofibrinosa dege nerans. Die verhältnismäßig geringgradige zellige Infiltration der Aderhaut ist nach beiden Auffassungen sekundärer Natur. -

Es er übrigt sich auf alle, besonders in der älteren Literatur unter fremden Namen und anderen Deutung zirkulierende Fälle näher einzugehen, da diese in den Arbeiten von Coats und Leber schon eine Berücksichtigung erhalten haben. Eher müssen diese Arbeiten herangezogen werden, welche nach der grundlegenden Veröffentlichung von Coats erschienen sind, besonders weil diese in manchen Hinsichten interessante Befunde und Schlüsse enthalten.

Hajanos ${ }^{2}$ ) Fall betraf einen 2 jährigen Knaben, bei welchem schon vor einem $1 / 2$ Jahre ein weiBer Schein aus der Pupille bemerkt wurde. Klinisch sah es wie Gliom aus. Die histologische Untersuchung zeigte, daß die Netzhaut durch eine eiweißreiche, zerfallene rote Blutkörperchen enthaltende Flüssigkeit abgelöst war. Die Netzhant war stark gefaltet in den Außenschichten. Wesentliche Veränderungen betrafen die Körnersehichten, wo ausgedehnte Degeneration und ein unregelmäßiges Lückensystem bestanden. Ganglienzellen waren ausgesprochen degeneriert. In dem vorderen Gebiet der Netzhaut waren große Blutungen und große

1) Leber, Graefe-Saemisch, Handb. d. Augenheilk., II. Aufl., y, 1267-1319.

2) Hajano, Ein Beitrag zur Kenntnis der Ret. exs. (Coats). Dieses Archiv 84, 30. 1913 . 
von Fibrin ausgefülte Hohlräume auf der Innenseite der Netzhaut. Gefäße stark verdickt, die endotheliale und adventitielle Wandverdickung führte zu fast völliger Obliteration, die Gefäße erschienen dadurch als ,knorrige Baumäste". Die Aderhaut war verdünnt und geringgradig zellig infiltriert. v. Hippel s ${ }^{1}$ ) Patientin war 49 Jahre alt, und die Enucleation wurde zwei Jahre nach Beginn des Leidens ausgeführt. Die klinischen Zeichen der Erkrankung gingen durch die Glaskörpertrübungen verloren. Außer dem Alter der Patientin ist abweichend, daß die Herde nicht in der Umgebung der Papille, sondern peripher gelegen sind. Er konnte große chorioretinitische Herde nachweisen, weiter subretinal mächtige Bindegewebsentwicklung. An der AuBenfläche der Netzhaut hafteten vascularisierte Schwarten, in die Retina drangen bindegewebige Stränge ein. An der Innenseite der Netzhaut Membranen, im Glaskörper Gefäßneubildung. Im Falle von Ginsberg und Spir $\mathrm{o}^{2}$ ) entwickelte sich bei einer 14 jährigen Patientin aus einer Neuritis nervi optici innerhalb 3 Wochen ein tumorförmiges Gebilde, binnen zwei Jahren eine Ablatio retinae, in weiteren zwei Jahren traten Glaukomanfälle auf, und nach sechsjährigem Bestehen des Leidens kam es zur Enucleation. Am anderen Auge ein großer, gelblichroter Knoten, der durch Jahre unverändert blieb. Die morphologisch als Tumor zu bezeichnende Neubildung bestand aus gewuchertem Gliagewebe und aus Blutgefäßen. Zwischen gliomatösen und angiomatösen Teilen bestand keine scharfe Abgrenzung. Das Wuchern der Blutgefäßle wird tumorartig angesprochen und ebenfalls die gliöse Wucherung, weil diese innerhalb des Tumorbezirkes zu hochgradig gewesen, um reaktive Vermehrung der Glia darzustellen. Nur die cystoide Gliose in der Ungebung des Tumors mit Gefäßneubildungen konnte reaktiver oder entzündlicher Herkunft sein. Ob der von Axenfeld ${ }^{3}$ ) mitgeteilte Fall sicher hierher gehört, muB fraglich erscheinen, schon deswegen, weil das Leiden doppelseitig einen 63jährigen Mann betroffen hat, bei dem an einem Auge zuerst als objektive Veränderungen kleine gelbliche Punkte in der Maculagegend auftraten, gefolgt von Hämorrhagien, und innerhalb $1 \frac{1}{2}$ Jahren nach der ersten Untersuchung war eine prominente, glänzende Masse hinter den Retinalgefäßen, von kleinen Blutungen umrahmt. Es wurde an Sarkom gedacht und enucleiert. Zwei Jahre später entwickelte sich aber auch am anderen Auge ein ähnlicher Prozeß, welcher aber zu einem der Vernarbung gleichenden Bilie fübrte. Im Bereiche dieses Pseudotumors war eine bindegewebige Masse, deren Vorderseite mit der Netzhaut zusammenhing und deren Rückseite der Aderhaut anlag. Die Außenschichten der Netzhaut sind aufgegangen in der bindegewebigen Masse, in welcher homogene kernarme Schichten mit kernreicheren und gefüBhaltigen abwechselten. Die Vascularisation der Schwarte besorgte die Choriceapillaris, die Capillaren der Zwischenkörnerschicht waren daran nur unwesentlich beteiligt. Das gewucherte Pigmontepithel schien in der Schwartenbildung beteiligt zu sein. Die nervösen Elemente der inneren Netzhautschichten waren degeneriert, das Stützgewebe stark gewuchert. Jedoch waren die Veränderungen der Netzhaut nicht wie die der Aderhaut sogesagt nur im Tumorbezirke. sondern das Neuroepithel war auch peripher hochgradig degeneriert. Weder Blutungen noch eisenhaltiges Pigment konnten nachgewiesen werden. Die Fälle, welche in der sehr genauen und lesenswerten Arbeit von Berg ${ }^{4}$ ) beschrieben wurden, gehörten alle einseitig erkrankten

1) v. Hippel, Anat. Befund bei einem Falle von Ret. exs. (Coats). Dieses Archiv 86, 443. 1913.

2) Ginsberg und S piro, Über Angiogliomatosis retinae (sog. v. Hippelsche Krankheit). Dieses Archiv 88, 44. 1914.

3) Axenfeld, Retinitis exs. cxt. mit Knochenbildung usw. Dieses Archiv 90, 452. 1915.

4) Berg, Beitr. zur path. Anatomie der Ret. exs. ext. Dieses Archiv 98, 211. 1918. 
jungen Leuten an. In drei Fällen war gleichfalls wie in unserem Falle wegen Gliomverdacht enucleiert worden. In sämtlichen Fällen lag eine herdförmige Degeneration der Netzhaut mit Gefäßveränderungen, Thrombosen und Aneurysmenbildung vor. Außerdem war eine ausgesprochene Gliawucherung, Bindegewebsneubildung in der Netzhaut, bindegewebige Neubildung zwisehen Netzhaut und Aderhaut, Ablösung der Netzhaut, rege Proliferation und Desquamation der Pigmentepithelien und Bildung von Fettkörnchenzellea zu verzeichnen. Sein Fall 3 ist Frühstadium, Fall 2 etwas älter, Fall 1 und 4 sind weit vorgeschrittene Stadien der Erkrankung. Emanuel ${ }^{1}$ ) hatte bei einem zweijährigen Knaben Netzhautablösung, Blutungen, Exsudation mit Cystenbildung, weitgehende kolloide Degeneration der äußeren Schichten und Bildung von homogenen Auflagerungen im Subretinalraum besehrieben. Die Retinalveränderungen entsprechen diesen Punkten, an denen eine kolossale Anhäufung von großen Gefäßen in den Innenschichten saß. Außerdem war eine zweifellos angiomatöse Neubildung des Ciliarkörpers im Zusammenhange mit den Geläßen des Ciliarkörpers vorhanden. Aus Hanssens ${ }^{2}$ ) Fällen gehört nur der Fall 2 in unsere Gruppe, in welchem bei einem 4.jährigen Kind die Netzhaut durch anscheinend feste tumorartige Massen vorgedrängt war. In dex anliegenden nasalen Netzhauthälfte waren große Veränderungen, hauptsächlich wirres gliöses Gewebe mit Körnerresten. Stäbchen und Zapfen waren nirgends zu erkencen; multiple kleine Verwachsungen zwischen Netzhaut und Aderhaut, an letzteren Stellen fehlte das Pigmentepithel. In den Spalträumen zwizchen den Verwachsungsstellen lagen zu Haufen angeordnet abgestoßene, degenerierte und gewucherte Pigmentepithelien. Rundherum im Bulbus war zwischen Netzhaut und Aderhaut eine verschieden breite Sehwarte chorioidealen Ursprungs. Ausgedehnte Nekrosen. Gliawucherung, cystoide Entartung und mit dem sub. retinalen Raum kommunizierende und mit fibrinösem Exsudat erfüllte Hohlräume. Die Veränderungen waren nicht herdförmig, sondern diffus, auch bestanden entzündliche Veränderungen des vorderen Abschnittes. Der Fall von Friedenwald $^{3}$ ) ist durch das Vorkommen von ungemein zahlreichen miliaren Gefäßekta. sien, hauptsächlich venösen Charakters, gekennzeichnet.

Die erste Frage, welche beantwortet werden muß, ob unser Fall in diese Gruppe gehört, trotzdem daß er in manchen Hinsichten Abweichungen zeigt von diesen Befunden, die als charakteristische hingestellt wurden.

Wir glauben die Frage bejahen zu können und glauben, daß diese Unterschiede dadurch bedingt sind, daß der vorliegende Fall eben ein sehr frühes Stadium der Erkrankung darstellt.

Das Auftreten der Erkrankung, die Schmerzlosigkeit, das Fehlen jeglicher Entzündungserscheinungen, die Einseitigkeit des Leidens, das jugendliche Alter des Patienten, das Verborgensein des Leidens (nur durch den Strabismus wurden die Eltern veranlaßt, eine fachärztliche Untersuchung vornehmen zu lassen) bestärken uns in der Richtigkeit unserer Diagnose. Es wurde schon Frwähnung getan, daß das

T) Emanuel, Dieses Archiv 90, 344. 1915.

2) Hanssen, Drei Fälle von Pseudotumor des Auges, mit Beiträgen seltener Befunde usw. Klin. Monatsb]. f. Augenheilk. 65, 703. 1920. 1914.

$\left.{ }^{3}\right)$ Friedenwald, Transactions of the American Ophthalm. Soc. 13, 821. 
Leiden meistens im jugendlichen Alter beobachtet wurde und einseitig blieb. Die in der neueren Literatur mitgeteilten Fälle ergaben ähnliche Angaben. So war $\mathrm{Hajanos}$ Patient 2 Jahre alt, Berg konnte vier Fälle mitteilen, in welchen das Alter unter 15 Jahren (ein Patient war 2 Jahre alt) blieb, weiter betrug das Alter im Falle von Ginsberg und Spiro 14, im Falle Emanuel 2, im Falle Hanssen 4 Jahre. In sämtlichen war das Leiden gleichfalls einseitig. Nur im Falle von Hippel und Axenfeld waren ältere Individuen erkrankt, und im letzteren Falle bot das Bild außer der Doppelseitigkeit so viel Abweichendes, daß es nicht sicher behauptet werden kann, daß die Erkrankung bestimmt in diese Gruppe gehört. Weitere klinische Merkmale, die in unserem Falle entscheidend waren, sind das multiple Auftreten und die Lokalisation am hinteren Pole. Die beigelegte Abbildung, welche bei der ersten Untersuchung angefertigt wurde, zeigt noch drei voneinander vollständig isolierte Herde in der Umgebung der Papille, welche im Verlaufe der Beobachtung anwachsen, konfluierten und eine ringförmige Vorwölbung verursachten. Besonders sei aber aus den klinischen Daten das Fehlen von. Blutungen hervorgehoben. $\mathrm{Ob}$ es im späteren Verlaufe zu solchen gekommen wäre, kann nicht entschieden werden. Wichtig ist nur, daß in solchen Anfangsstadien diese völlig vermißt wurden, und so bei der Differentialdiagnose des Leidens ihnen eine wichtige Rolle nicht zuerteilt werden kann. Möglich wäre es nach den pathologisch-anatomischen Gefäßveränderungen, daß später Blutungen entstehen, und darum evtl. Bilder resümieren können, wie im Falle von Axenfeld, der eben den Blutungen eine wichtige Rolle bei der Diagnosenstellung zuweist.

Noch charakteristischer war der mikroskopische Befund gewesen. Die minimale Infiltration in der Aderhaut, die eingeschobene Zwischenschwarte, die hochgradige Fältelung der Netzhaut sind alle Veränderungen, die sich in den Befunden der verschiedenen Autoren wiederholen. Die geringgradige zellige Emigration in der Aderhaut scheint ein konstantes Symptom zu sein. Nur wenige Fälle waren, welche ein anderes Verhalten zeigten. Im Falle von Hi p pel werden die hochgradigen Veränderungen der Aderhaut mit einer mächtigen exsudativen Chorioiditis identifiziert, neben welche eine selbständige Retinitis herangezogen wird. Dabei bleibt die Möglichkeit einer koordinierten Entzündung beider Membrane oder die sekundäre Entstehung in einer der Membrane offen.

Die Veränderungen der Netzhaut variiert schon in den beschriebenen Fällen zu stark. Diese können ohne weiteres auf den Umstand zurückgeführt werden, daß eben selten Frühstadien der Erkrankung untersucht wurden, und daß in den Spätstadien schon die mannigfaltigsten Veränderungen auftreten können. Aber in der Beurteilung der primären 
Veränderungen können nur Jugendstadien verwertet werden. Dieser Umstand verleiht unserem Falle eine ganz besondere Wichtigkeit und erklärt auch, warum z. B. Blutungen, welchen Coats so eine ausschlaggebende Rolle zugesprochen hat, völlig vermißt wurden.

Zweifellos sind die frühesten Veränderungen die Verdickung, Fältelung und das Adhärentsein der Netzhaut, welche in unserem Falle die tumorartige Vorbuckelung der Netzhaut vortäuschten. Außer diesen waren Gefäßwandveränderungen, mäßige gliöse Wucherung und Degeneration der Ganglienzellen, Höhlenbildung, fibrinöse Exsudation in den innersten Schichten und in den Einbuchtungen und schlauchartigen Kcnfigurationen der äußeren Schichten, weiter ein zell- und gefäßreiches Zwischengewebe zwischen Netzhaut und Aderhaut vorhanden. - Die ersten drei Veränderungen: Verdickung, Fältelung und Verwachsung beider Membranen werden allgemein als früh einsetzende angesehen. Coats nimmt aber Blutungen in den äußeren Schichten, Leber dagegen ausges prochene Nekrose infolge tiefgreifender Ernährungsstörung an. In unserem Falle wurden beide vermißt, dagegen beherrschten die Gefäßveränderungen, auf welche wir noch zurückkommen, das Bild. Es will uns daher scheinen, daß beide Veränderungen, die Blutungen und dienekrotischen Veränderungen, gleichfalls nicht das Primäre darstellen, viel eherzu den späteren Folgeerscheinungen einzureihen sind. Als spätere Folgeerscheinungen dürfen beide als typische gelten, jedoch dürfen eben nur obige Ursachen nicht mit der Erkrankung in kausalen Zusammenhang gebracht werden. Solche ty pische Folgeerscheinungen sind mehrere bei diesen Leiden bekannt, welche gleichfalls in unserem Falle noch nicht aufgetreten sind. So treten im späteren Verlaufe der Erkrankung die Gespensterzellen (Coats) auf, bei welchen mit Hilfe der Sudanfärbung Leber fettige Degeneration nachgewiesen hat und aus diesem Grunde sie Fettkörnchenzellen nannte und aus den Pigmentepithelien ableitete. Die Exsudation und die dadurch bedingte Abhebung der Netzhaut müssen auch späteren Stadien angehören, nachdem in unserem Falle wohl eine Adhäsion, jedoch keine Abhebung, auch kein Erguß im subretinalen Raum vorhanden gewesen waren. Es erübrigt sich auf sämtliche Veränderungen im späteren Verlaufe näher einzugehen, so auf die Abkapselungen, Einwanderungen der Fettkörnehenzellen in die Netzhaut, auf das Auftreten von Cholesterin usw., welche allgemein bekannt sind, und außerdem in unserem Falle war der Prozeß nicht so weit gediehen, daß ähnliche pathologische Veränderungen zu verzeichnen gewesen wären.

Desto wichtiger erscheinen uns die Veränderungen der Gefäße, in welchen die Mögliehkeit der fibrinösen bzw, serofibrinösen Durch- 
tränkung der Netzhaut, weiter die der Degeneration erblickt werden muß. Berg konnte in seinen Fällen gleichfalls schwerste Veränderungen der Gefäße nachweisen, welche durch Thrombosen zu Nekrosen führten und von Berg auch für primäre Veränderungen hingestellt wurden. Bei der Beschreibung wurde es klar gelegt, daß die Gefäße verschiedene Veränderungen, jüngerer und älterer Herkunft, aufgewiesen haben, welche nebeneinander angetroffen wurden, ebenso wie die Veränderungen der Intima- und Adventitialzellen. Von der zungenförmigen oder ringförmigen Wucherung der Endothelien waren alle Übergänge bis zum vollständigen Verschluß des Lumens reichlich vertreten. Auch

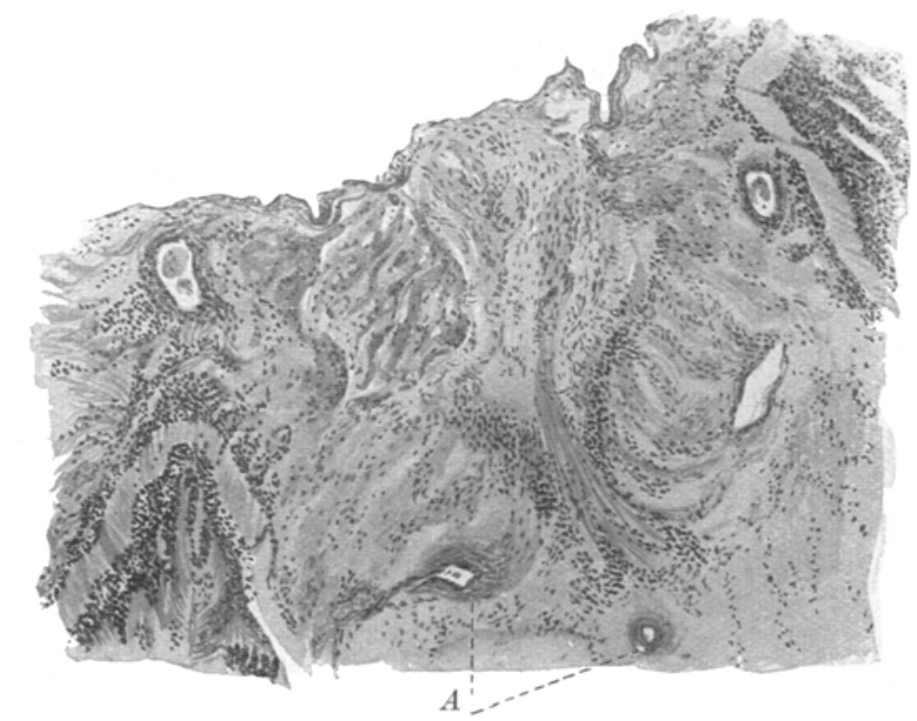

Abb. 5. Zentrale Teile der Papille. $A=$ ringtörmige Wucherungen der Adventitialzellen.

auf die mantelartigen Wucherungen der Adventitialzellen wurde hingewiesen, und daß letztere auch vom eigentlichen Herde weiter entfernt sichtbar waren. Berg erblickt auch in den Wucherungen des Endothels und in der Rundzelleninfiltration der Wandung die primäre Veränderung, die Hyalinisierung, Erweiterung und Aneurysmenbildung sollen schon auch spätere Stadien darstellen. Berg betont besonders die Bildung der Aneurysmen, welche Leber auch nicht entgangen sind und eben zu der Abgrenzung einer besonderen Erkrankung bei Jugendlichen den Anlaß gegeben hat. In dem Fall 2 von Berg konnte die Bildung der Aneurysmen genau verfolgt werden; Rupturen der GefäBwand, Austritt und Abkapselung der Blutelemente durch die Adventitialelemente, Sprengung der letzteren markieren den Vorgang und führen zum Austritt des Blates in die Netzhaut. Außer Miliar- 
aneurysmen waren auch dissoziierende Aneurysmen vorhanden. Unser Fall stellt aber ein noch jüngeres Stadium dieser Veränderungen vor. Wahrscheinlich wären später ähnliche Veränderungen aufgetreten, welche selbstredend in hohen Graden zur Thrombosenbildung und pathologischen Umgestaltung der Strömungsverhältnisse führen und somit außer den eigentlichen Wandveränderungen das Auftreten von Blutungen begünstigen. Bemerkenswert ist auch der Fall v. Hippel in dieser Beziehung, wo in einigen Schnitten in der Zentralarterie eine aus Endothelzellen gebildete Masse lag, in welcher aber auch Kerne der Muscularis nachweisbar waren. Letztere veranlaßten v. Hip pel anzunehmen, daß die gegenüberliegende Wand der Arterie das Bild der Proliferation vortäuschte, Le ber führt a ber diesen Befund als endarteriitische Wucherung an. Coats und Le ber haben teilweise in ihren Fällen gleichfalls Veränderungen der Gefäße notiert. Doch sie faßten es nicht als primäre auf, weil es in manchen Fällen vermißt wurde oder weil der Ort der Gefäßerkrankungen mit dem Orte der hauptsächlichen Veränderungen der Netzhaut nicht kongruierte. Und so sah sich Coats veranlaßt, diese durch die Staung mechanisch hervorgerufen zu erklären (Schrumpfung und Zirkulationsstörung im Gebiete des primären Netzhautherdes). - Alle diese Befunde bezogen sich mit Ausnahme von unserem auf Veränderungen bzw. Proliferation der Endothelien. Wir waren aber in der Lage gewesen, ähnliche Vorgänge auch in der Adventitia nachweisen zu können, Veränderungen, die nur im Befunde von Ginsberg und Spiro erhoben wurden, von diesen Autoren aber für ihren Fall diese Erklärung nicht annehmbar gewesen, nachdem die Einwanderung der höchstgradig gewucherten Elemente der Glia nicht auszuschließen war. -

Zweifellos zeigt sich also, daß in Frühstadien der Erkrankung, und so auch in unserem Falle, die hochgradigen Veränderungen der Gefäße vorangeben und das Primäre darstellen. Eben diese bedingen völlige Verstopfungen, Aneurysmen, Blutungen, welche geeignet sind, das ursprüngliche Bild zu verschleiern oder vollständig zu ersetzen. Vielleicht findet der Umstand, daß eben in späteren Stadien, wo große Blutungen in der Netzhaut auch subretinal gelegen sind, die Gefäßveränderungen nicht nachweisbar gewesen sind, in dieser Annahme ihre Klärung. - -

Außer Gefäßveränderungen war in unserem Falle die Fältelung, die Bildung der Zwischenschwarte und fibrinöse Exsudation vorhanden. Alle anderen Veränderungen, welche beschrieben wurden, fehlten, und so gehören diese keinesfalls dem Jugendstadium an. -

Was die Aufeinanderfolge der einzelnen Symptome anbelangt, so soll außer der Coats- bzw. Leberschen Erklärung nur das Wichtigste kurz gestreift werden. - 
Hajanos Ansicht nach spräche die Einseitigkeit des Leidens für die lokale Entstehung, zwar nicht bezwingend, weil bei Allgemeinerkrankungen einseitige Augenerkrankungen ebenfalls auftreten können. Faßt es als degenerative Erkrankung auf, bei welcher Gefäßerkrankungen mit multiplen Blutungen und Netzhautdegenerationen Hand in Hand gehen, doch scheint die Gefäßalteration des Primäre zu sein. Ginsberg und Spiro nehmen multiple, aus Glia und Blutgefäßen in wechselnder Zusammensetzung bestehende Tumoren an, nennen es „Angiogliomatosis retinae und rechnen es zu der Gruppe der v. Hippelschen Angiomatosis retinae. Emanuel nimmt aber eine multiple primäre Angiomatose als bewiesen an, lehnt die Mellersche Auffassung der Gliomatosis ab, und ebenso die Möglichkeit der gleichzeitigen Beteiligung der Glia und Gefäße an der Geschwulstbildung. Im erwähnten v. Hippelschen Fall fehlten die Blutungen, und das anatomische Bild ergab eine echte entzündliche Chorioretinitis. Hanssen nennt die Veränderungen der äußeren Schichten primär, während die bindegewebigen Neubildungen an der Rückseite und das Exsudat im subretinalen Raum hinter der abgehobenen Netzhaut toxische Reizerscheinungen wären. -

Die Verschiedenheit dieser Auffassungen beruht wohl auf dieser Grundlage, daß im Spätstadium das Bild selbst von sekundären Veränderungen stark verunstaltet wird und so die Deutung der einzelnen Erscheinungen auf große Schwierigkeiten stößt. So wird es auch erklärlich, daß die Autoren andere Fälle zu den ihrigen rechnen, oder umgekehrt aus diesen ausschließen. So z. B. wird der Fall von Emanuel von Berg in die Gruppe der Retinitis exsudativa eingeteilt, dagegen der Fall Axenfeld eher einer selbstänỏigen, bei älteren Individuen vorkommenden Gruppe zugeschrieben. Besonders häufig sind die Verschiebungen zwischen der $v$. Hippelschen Angiomatosis retinae und Retinitis exsudativa (Coats), was eher verständlich ist, weil es in Spätstadien bei der v. Hippel schen Erkrankung zu massiger Exsudation kommen kann, und dementsprechend auch bei der Coatsschen Erkrankung Veränderungen sich manifestieren können, welche geeignet sind, die Grenzen zwischen beiden zu verwischen.

Es mag vorkommen, daß in einem gegebenen Falle die klinische Untersuchung, besonders in älteren Fällen, nicht die sichere Unterscheidung bringen kann. Die anatomische Untersuchung, insbesondere die der Jugendstadien, erfordert eher die schärfste Trennung und Absonderung. Das Wesen und die Genese der v. Hippel schen Erkrankung ist grundverschieden gegenüber unserer Erkrankung, es besteht aus einer Angiomatose der Netzhaut, an welcher sich auch die Glia beteiligen kann und welche auch von entzündlichen Erscheinungen später gefolgt werden kann. - 
Was ist demgegenüber die Retinitis exsudativa externa Coats bzw. serofibrinosa degenerans (Leber)?

Bei Beantwortung obiger Frage sei daran erinnert, daß die Pathoge nese nur an frischen Fällen zu unterseheiden ist, und daß die meisten Fälle solche Spätstadien darstellen, in welchen das Primäre vom Sekundären schwer abgrenzbar war, oder wurden die primären Erscheinungen überhaupt gedeckt. Nur wenige Fälle können es beanspruchen, bei dieser Frage herangezogen zu werden. Eine Ausnahme bildet diesbezüglich der Fall I von Coats und der Fall III von Berg. Und eben in diesen verhältnismäßig frischen Stadien waren die Gefäßveränderungen sehr markant. Coats erwähnt in der Beschreibung seines Falles, daß die Gefäße zu 2 bis 3 zusammenlagen und kavernösen Knötchen glichen, manchmal zeigte die Wandung eine rundzellige Infiltration ohne Verdickung. Die mittleren und kleineren Gefäße waren verdickt und von hyaliner Degeneration betroffen, welche in den kleinsten Gefäßen sogar zu Obliterationen führte. An einigen Stellen waren die Gefäße so zahlreich, daß eine Gefäßneubildung angenommen wurde. Im früher erwähnten Falle von Berg waren die Endothelien der GefäBe vielfach gewuchert und gequollen, weiterlag Rundzelleninfiltration und schwache. Hyalinisierung der Wandung vor.

Diese zwei Fälle zeigten die ausgesprochen ähnlichen Veränderungen

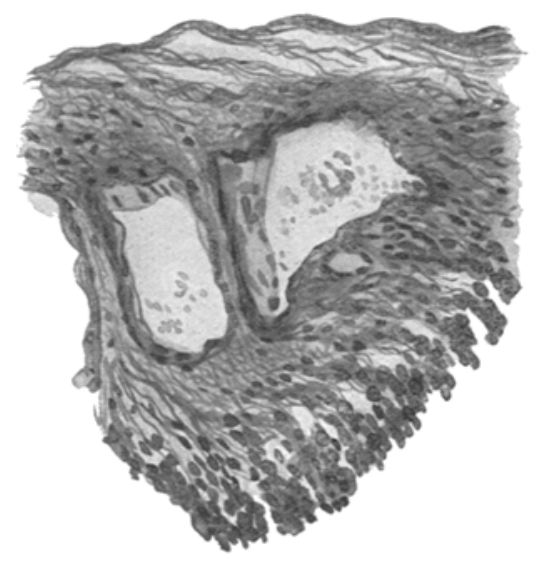

Abb. 6. Partie aus den inneren Netzhautschichten. Deutliche Proliferation der Hndothelzellen mit Verengang des Iumens.

der Gefäßwandung wie unser Fall. Dabei ist die Beweiskraft dieser Fälle nicht so zwingend, weil diese gewiß ältere Stadien repräsentieren als unser Fall. Bei Berg war es doch schon zu völliger Netzhautabhebung und Aufrollung derselben gekommen mit großen Blutungen älterer und jüngerer Herkunft, im Falle von Coats waren mit Blutkörperchentrümmern und Cholesterin erfüllte Hohlräume doch anwesend. Die Durchsicht der einschlägigen Fälle scheint uns zu zeigen, daß dieser Fall das jüngste Stadium der untersuchten Fälle vertritt, wo Netzhautabhebung, subretinales Exsudat, Blutungen, Höhlenbildung (letztere nur angedeutet), Fettkörnchenzellen, hochgradige Gliaw ucherung noch nicht zur Ausbildung gekommen waren. Das völlige Fehlen der Folgeerscheinungen bekräftigt die Wichtigkeit der vorhandenen Veränderungen und zeigt an, welche Veränderungen das 
Primäre und Charakteristische der Erkrankung ausmachen. Die hau ptsächlichen Veränderungen waren: l. Ausgebreitete Gefäßveränderungen verschiedenen Grades, welchen in erster Linie die kleineren und kleinsten Gefäße unterlagen, und stellenweise vollständig obliterierten. Außer der Wucherung der Endothelien sei nochmals auf die nachgewiesenen Veränderungen der Adventitialzellen hingewiesen. 2. Eine serofibrinöse Durchtränkung der Netzhaut und Ablagerung vom Exsudate vorwiegend in den inneren Schichten sind an der Außenfläche bzw. zwischen den Falten der äußeren Netzhautschichten. 3. Die Bildung einer mit der Netzhaut anscheinend festverbundenen zell- und gefäßreichen Zwischenschicht zwischen Aderhaut und Netzhaut, mit Einwanderung der gew ucherten Pigmentepithelien der Aderhaut. - Alle ïbrigen vorhandenen Veränderungen waren zu geringgradig, um ihnen eine wesentliche Rolle erteilen zu können. So verhält es sich mit der spärlichen Infiltration in der Aderhaut; die mäßige Gliawucherung besonders der inneren Schichten, weiter die aus einzelnen roten Blutkörperchen bestehenden Hämorrhagien sind gewiß von untergeordneter Bedeutung. -

Wenn wir den ganzen Werdegang des Prozesses zu verbildlichen versuchen, so können nur die Veränderungen der Gefäße den Ausgangspunkt liefern, aus welchen sich alle Frscheinungen mit Wahrscheinlichkeit und Leichtigkeit ableiten lassen. Die Vorstellung, daß solche Gefäßveränderungen infolge Zirkulationsstörungen zu serofibrinöser Durchtränkung und zu Blutungen Anlaß geben können, bedarf ja keiner besonderen Beweise. Berg, der m. E. die primäre Beteiligung der Gefäße richtig hervorhob, hat das Wesentliche des Prozesses herausgegriffen. Die Blutungen, Nekrosen, Degenerationen verdanken ihre Entstehung nur den bereits gegebenen Gefäßveränderungen. Coats's und Lebers Aufmerksamkeit entgingen die Gefäßveränderungen gleichfalls nicht. Doch verlegte Coats den Schwerpunkt auf die Blutungen und leitete aus diesen die übrigen Veränderungen ab. Die Gründe dafür sind darin gegeben, daß seine Fälle eben sehr späte Stadien darstellen, wo eben die Aufeinanderfolge der einzelnen Veränderungen nicht genau zu bestimmen ist. In seiner Auffassung entsteht die Verwachsung, die Zwischenschicht auch in den späteren Stadien, dagegen konnten wir zeigen, daß im Anfangsstadium, wo Blutungen vollständig fehlten, schon diese Schwarte ausgebildet war. Leber argumentierte gegen die primäre Rolle der Gefäße damit, daß diese nicht immer nachweisbar wären. Doch kann das Fehlen der Gefäßwandveränderungen in späteren Stadien dadurch bedingt sein, daß durch Obliteration der Gefäße und gleichzeitiges Überwuchern von den sekundären Erscheinungen diese gedeckt 
oder verwischt werden, wenn auch $\mathrm{Hanssen}$ gegen diese Auffassung sein Bedenken ausspricht. -

Die Richtigkeit dieser Deutung scheint nun auch die Beobachtung von Hegner noch zu bekräftigen, trotzdem, daß dieser Fall nicht in jeder Beziehung verwertet werden kann. Hegner ${ }^{1}$ ) hat bei einem 70 jährigen Manne eine doppelseitige Affektion im Gefolge einer Lymphogranulomatose beobachtet, welche als Retinitis exsudativa aufgefaßt wird. Selbst die Doppelseitigkeit, das Auftreten im späteren Alter bei Lymphogranulomatose macht Bedenken, ob dieser Fall mitgerechnet werden darf oder eine Erkrankung darstellt, welche erst später von unserer Gruppe genau isoliert werden wird. Der mikroskopische Befund hatte tatsächlich vieles, welches die Diagnose stützt. Für unsere Frage hat es aber ein ganz spezielles Interesse, nachdem in diesem nicht ganz typischen Falle (perivasculäre Herde in der Aderhaut, Netzhaut und in der Orbita) Gefäßveränderungen beobachtet wurden, welche aus den adventitiellen Zellen ausgegangen, die Gefäßwand destruierten und in das Lumen übertraten. Wenn wir auch annehmen, daß dieser Fall nicht eine echte Retinitis exsudativa wäre, sondern da $\beta_{3}$ als Teilerscheinung der Lymphogranulomatose ein unserer Erkrankung ähnlicher Prozeß vorgelegen wäre, so zeigt er doch, daß die adventitiellen Zellen durch Proliferation weitgehende Veränderungen hervorrufen können und daß diese entzündliche Proliferation ein Krankheitsbild erzeugen kann, welches, wenn nicht identisch, doch dem unserem auffallend ähnlich ist. -

Alles in allem ist die Erkrankung eine rein entzündliche, wobei die Veränderungen der Gefäße ein ausschlaggebendes Moment darstellen. Das Bild des Jugendstadiums berechtigt diese Annahme und zwingt uns zu der Ableitung der Symptome der späteren Stadien aus dem Vorhergesagten. Außer Gefäßveränderungen waren serofibrinöse Durchtränkung und die Bildung einer zellreichen Schwarte zu verzeichnen. Somit erübrigt sich hervorzuheben, daß diese von der Angiomatosis retinae (v. Hi p pel) völlig abweicht, und nur die unspezifischen Späterscheinungen mögen das klare Bild dermaßen überdecken, daß eine Verwechslung ermöglicht wird.

Wenn wir den Erscheinungskomplex der Entzündung auf breiter Grundlage analysieren, so sehen wir regressive und progressive Ernährungsstörungen neben vasculären Störungen. Diese Trennung der Symptome veranlaßte $L u b a r s c h$ dazu, daß er die einheitliche Gruppe in alternative, exsudative und produktive Entzündungen auflöste, je nachdem in dem bestimmten Falle die eine oder andere der drei genannten Komponenten in dem ganzen Erscheinungskomplex überwiegt. Dieser

i) Hegner, Retinitis exsudativa bei Lymphogranulomatosis. Klin. Monatsbl. f. Augenheilk. 5\%, 27. 1916. 
996 A. Rados: Über die Veränderungen im Frühstadium der Retinitis usw.

Einteilung folgend wäre in unserem Falle eine Mischung von vasculären und progressiven Ernährungsstörungen vorhanden. Da aber diese Trennung, das quantitative Übergewicht des einen oder anderen Komplexes berücksichtigend, nicht volle Berechtigung beanspruchen kann, sehen wir ab, diese für die Benennung als ausschlaggebend zu akzeptieren. Es soll nun hervorgehoben werden, daß bei dieser Art Entzündung der Netzhaut in erster Linie vasculäre Störungen und in zweiter Linie progressive Ernährungsstörungen das Übergewicht haben. Die regressiven Ernährungsstörungen treten nur in späteren Stadien dieser Entzündung in den Vordergrund, vergesellschaftet mit Erscheinungen, welche schon überhaupt nicht zum primären Erscheinungskomplex der Entzündung gezählt werden können. 